\title{
Quantum Physical Systems and Their Evolution
}

\author{
Malkhaz Mumladze \\ Gori University, Gori, Georgia \\ Email: mmumladze@mail.ru
}

How to cite this paper: Mumladze, M. (2018) Quantum Physical Systems and Their Evolution. Open Access Library Journal, 5: e4244.

https://doi.org/10.4236/oalib.1104244

Received: December 11, 2017

Accepted: January 8, 2018

Published: January 11, 2018

Copyright $\odot 2018$ by author and Open Access Library Inc.

This work is licensed under the Creative Commons Attribution International License (CC BY 4.0).

http://creativecommons.org/licenses/by/4.0/

\section{(c) (i) Open Access}

\begin{abstract}
In this article, we proposed a method for describing the evolution of quantum physical systems. We define the action integral on the functional space and the entropy of distribution of observable values on the set of quantum states. Dynamic of quantum system in this article is described as dynamical system represented by one parametric semi group which is extremal of this action integral. Evolution is a chain of change of distribution of observable values. In the closed system, it must increase entropy. Based on the notion of entropy of distribution energy, on the principle of maximum entropy production, we get a picture of evolution of closed quantum systems.
\end{abstract}

\section{Subject Areas}

Modern Physics, Quantum Mechanics

\section{Keywords}

Quantum Physical System, State, Pure State, Observable, Measure, Probability Measure

\section{Introduction}

A quantum physical system can be represented by a couple $(U, \mathfrak{I})$, where $U$ is some $C^{*}$-algebra Hermitian elements of which are called observables and $\mathfrak{I} \subset E_{U}$ is some subset of the set $E_{U}$ positive functionals with norm one called as the set of quantum states of this physical system. For the description of the evolution of quantum systems, one-parameter semi groups, groups and different operators and differential equations which are derived from different variance principles have long been used. In this work, we will try to construct a variational problem to find one-parameter semi groups describing the evolution of a quantum system [1]-[6].

Denote by $P_{U}$, the set of pure states on $C^{*}$-algebra $U, P_{U} \subset E_{U}$. 
In the set of linear, continuous functionals on the $C^{*}$-algebra $U$, we have the topological structure which is called as ${ }^{\star}$ weakly topological structure and which is defined by pre base:

$$
V\left(\omega, u_{1}, u_{2}, \cdots, u_{n}\right)=\left\{\omega^{\prime} \in U^{*}|| \omega\left(u_{i}\right)-\omega^{\prime}\left(u_{i}\right) \mid<\varepsilon, i=1,2, \cdots, n\right\},
$$

where $V, \omega, \omega^{\prime} \in U^{*}, u_{i} \in U \quad[2]$, and accordingly this in the set $P_{U}$ we have the topological structure induced from this topological structure .

Denote by $\mathfrak{R}$ the set of Hermit's elements of $U \quad C^{*}$-algebra.

Let $\left\{p_{\alpha}\right\}$ be the set of all one-dimensional projectors on $C^{*}$-algebra $U$ and $\omega$ pure state. In the workthi [7], we showed that pure state has the meaning 1 only on one one-dimensional projector $p_{\alpha} \in\left\{p_{\alpha}\right\}$ and the meaning 0 on the other one-dimensional projectors. Denote pure state which on projector $p_{\alpha}$ has the meaning 1 as $\omega_{\alpha}$.

An integral representation of Hermit's elements $u \in \mathfrak{R}$

$$
u=\int_{-\infty}^{\infty} \lambda \mathrm{d} p_{\lambda}^{u}
$$

where $\lambda$ element of spectrum $\sigma_{u}$ and $p_{\lambda}^{u}$ element of partition of unity of Hermit's element $u \in \mathfrak{R}$ [8] follow that for the pure states $\omega_{\alpha}$ has placed the equality $\omega_{\alpha}(u)=\lambda_{\alpha}^{u}$, where $\lambda_{\alpha}^{u}$ is some element of spectrum of Hermit element $u \in \mathfrak{R}$. It gives opportunity to identify every pure state with the set of number $\left\{\lambda_{\alpha}^{u}\right\}_{u \in \mathfrak{R}}$, where $p_{\alpha}(u)=\lambda_{\alpha}^{u}$.

Consider the Tikhonov's product $\Sigma=\bigotimes_{u \in \Re} \sigma_{u}$, where $\sigma_{u} \subset R$ spectrum of element $u \in \mathfrak{R}$. It is clear that $P_{U} \subset \Sigma$, because $P_{U}$ is the set of such elements in product $\Sigma=\bigotimes_{u \in \Re} \sigma_{u}$ which represent continuous linear mapswhit respect to the topological structure in $\mathfrak{R}$ induced by norm from $C^{*}$-algebra $U$ :

$$
P_{U}=\left\{\omega_{\alpha}: \mathfrak{R} \rightarrow \underset{u \in \mathfrak{R}}{\cup} \sigma_{u} \mid \omega_{\alpha}(u)=\lambda_{\alpha}^{u}, \omega_{\alpha}\left(k_{1} u_{1}+k_{2} u_{2}\right)=k_{1} \omega_{\alpha}\left(u_{1}\right)+k_{2} \omega_{\alpha}\left(u_{2}\right)\right\} .
$$

Consequently in the set $P_{U}$, we have topological structure induced from Tikhonov's product $\Sigma=\underset{u \in \Re}{\otimes} \sigma_{u}$. This topological structure coincides with the induced topological structure from weakly topological structure on set of functionals on $C^{*}$ algebra $U$.

For every state $\omega \in \mathfrak{J}$ we have $\int_{-\infty}^{\infty} \mathrm{d} \omega\left(p_{\lambda}^{u}\right)=1$, therefore it is easy that the value of quantum state on observable $u \in \mathfrak{R}$ is the middle value of this observable. The value $\omega(u) \in R$ is called the middle (average) value of observable $u \in \mathfrak{R}$ of quantum physical system in the state $\omega \in \mathfrak{I}$.

Every state is an element of the closure of the set convex linear combinations of pure states in the ${ }^{\star}$ weakly topological structure [2]. Let us call in the space $P_{U}$ the closure of the set of pure states which generate $\omega \in E_{U}$ state by the support of this state and denote it as Support $\omega$.

If given physical quantum system $(U, \mathfrak{I})$, where each state $\omega \in \mathfrak{I}$ 
represents some elementary particle, this elementary particle corresponds to set of pure states, Support $\omega$. This means that elementary particles of system $(U, \mathfrak{I})$ located in subspace $P_{\mathfrak{I}} \subset P_{U}$, where

$$
P_{\mathfrak{\Im}}=\bigcup_{\omega \in \mathfrak{I}} \text { Support } \omega \text {. }
$$

Let us call the subspace $P_{\mathfrak{I}}$ by physical space of the physical quantum system $(U, \mathfrak{I})$.

Further, we denote the quantum physical system by $\left(U, P_{\mathfrak{I}}, \mathfrak{I}\right)$

\section{Dynamics of Quantum System}

Well known that the map $\pi: U \rightarrow U^{* *}$ defined by formula $\pi(u)(\omega)=F_{u}(\omega)=\omega(u)$ is isometric embedding $U$ as Banach space in the double conjugate space $U^{* *}$ [8]. If $\omega$ is a state then $\omega\left(u^{*} u\right) \geq 0$ [2]; it follows, that if $u \in U$ is positive element, then $u=v^{*} v, v \in U$ and $\omega(u) \geq 0$.

Thus, if $u \in U$ is positive element then $F_{u}(\omega) \geq 0$ for each state on $U$. Because $\pi$ is isometric, therefore $\left\|F_{u}\right\|=\|u\|$.

$$
\left(F_{u}+F_{v}\right)(\omega)=F_{u}(\omega)+F_{v}(\omega)=\omega(u)+\omega(v)=\omega(u+v)=F_{u+v}(\omega) .
$$

If $u$ is hermit's element $u=\int_{-\infty}^{\infty} \lambda \mathrm{d} p_{\lambda}^{u}$, because, for such elements $F_{u}=\int_{-\infty}^{\infty} \lambda \mathrm{d} F_{p_{\lambda}^{u}}$ and

$$
F_{u}(\omega)=\int_{-\infty}^{\infty} \lambda \mathrm{d} F_{p_{\lambda}^{u}}(\omega)=\int_{-\infty}^{\infty} \lambda \mathrm{d} \omega\left(p_{\lambda}^{u}\right) .
$$

We note that the middle (average) value of the observed $u \in U$ in the state may not enter into the spectrum of this observable. Therefore we can assume that $\mathfrak{I}$ is closed subset of Tikhonov's product $\Sigma=\otimes \bar{\sigma}_{u}$, where $\bar{\sigma}_{u}$ is the minimal closed interval which contains $\sigma_{u}$.

On each interval $\bar{\sigma}_{u}$ we have a Lebesgue measure $l_{u}$ and on $\underset{u \in \Re}{\otimes} \bar{\sigma}_{u}$ the product measure $\underset{u \in \mathfrak{R}}{\otimes} l_{u}[9]$ of this measures. The sets $\mathfrak{I}$ and $E_{U}$ measurable in $\underset{u \in \Re}{\otimes} \bar{\sigma}_{u}$ with measure $\underset{u \in \Re}{\otimes} l_{u}$.

In quantum physical system

$$
\left(U, P_{\mathfrak{I}}, \mathfrak{I}\right)
$$

for hermit's positive observable $u \in U$ the functional $F_{u}$ may be considered as distribution observable $u$ on the states $\omega \in \mathfrak{I}$.

Let $u_{E} \in U$ positive observable represents the energy of system $\left(U, P_{\mathfrak{I}}, \mathfrak{I}\right)$, then $F_{u_{E}}$ describes the density of distribution of energy in this system. If we assume that the time is the change distribution of energy in the system then the movement of our system we can describe by one-parameter continuous semi group

$$
\left\{d_{t} E_{U} \rightarrow E_{U}, t \in[0, \infty)\right\}
$$


where: $E_{U} \subset \Sigma$ set of all states, $d_{0}$ is identify map, $d_{s} \circ d_{t}=d_{t+s}$ where each parameter value $t$ corresponds to own distribution of energy $F_{u_{E}} \circ d_{t}$ i.e. to time moments.

Define the measures $\mu_{u_{E}}^{t}, t \in[0, \infty)$ on the set of states $d_{t}\left(\mathfrak{I}_{0}\right)=\mathfrak{I}_{t} \subset E_{U}$, where $\mathfrak{I}_{0}=\mathfrak{I}$. The map $F_{u_{E}} \circ d_{t}$ is continuous. It follows, that if $A \subset \mathfrak{I}_{t}$, where $A$ measurable with respect to measure $\underset{u \in \Re}{\otimes} l_{u}$ on $\mathfrak{I}_{t}$, then integral

$$
\mu_{u_{E}}^{t}(A)=\int_{A} F_{u_{E}}(\omega) \mathrm{d}\left(\underset{u \in \Re}{\otimes} l_{u}\right) .
$$

exist and defines new measure on $\mathfrak{J}_{t}$

Consider product measure $\underset{\substack{\otimes \\ t \in[0, \infty)}}{\otimes \mu_{u_{E}}^{t}}$ on $\underset{t \in\{0, \infty)}{\otimes} \mathfrak{I}_{t}$. This measure describes distribution of energy over the trajectories of the evolution of the system.

Let $\left\{\left\{d_{t}^{\alpha}: E_{U} \rightarrow E_{U}, t \in[0, \infty)\right\}\right\}_{\alpha \in \Gamma}$, the set all of one- parameter semi groups on $E_{U}$ For every this semi group we have measures on $\mathfrak{J}_{t}^{\alpha}=d_{t}^{\alpha}\left(\mathfrak{\Im}_{0}^{\alpha}\right), \mathfrak{I}_{0}^{\alpha}=\mathfrak{I}$ :

$$
{ }^{\alpha} \mu_{u_{E}}^{t}(A)=\int_{A} F_{u_{E}}(\omega) \mathrm{d}\left(\underset{u \in \Re}{\otimes} l_{u}\right),
$$

where $A \subset \mathfrak{J}_{t}^{\alpha}$ measurable with respect to measure $\underset{u \in \Re}{\otimes} l_{u}$ on $\mathfrak{J}_{t}^{\alpha}$. The product measures $\underset{t \in[0, \infty)}{\otimes} \mu_{u_{E}}^{t}$ on $\underset{t \in\{0, \infty)}{\otimes} \mathfrak{J}_{t}^{\alpha}$ describes distribution of energy over the trajectories of the evolution of the system when this evolution is described by one parametric semi group $\left\{d_{t}^{\alpha}: E_{U} \rightarrow E_{U}, t \in[0, \infty)\right\}$.

Let $A_{d_{t}^{\alpha}} \subset \underset{t \in\{0, \infty)}{\otimes} \mathfrak{J}_{t}^{\alpha}$ is set of trajectories of evolution of system $\left(U, P_{\mathfrak{J}}, \mathfrak{I}\right)$, described by semi group $\left\{d_{t}^{\alpha}: E_{U} \rightarrow E_{U}, t \in[0, \infty)\right\}$. Measure $\underset{t \in[0, \infty)}{\otimes} \mu_{u_{E}}^{t}\left(A_{d_{t}^{\alpha}}\right)$ represents the total energy contained in the set $A_{d_{t}^{\alpha}}$ of trajectories.

If $\bar{A}_{d_{t}^{\alpha}}$ the set of all trajectory of evolution of the system, then the measure $\underset{t \in[0, \infty)}{\otimes{ }^{\alpha}} \mu_{u_{E}}^{t}\left(\bar{A}_{d_{t}^{\alpha}}\right)$ we will consider as the total energy contained in the set of all trajectory of evolution quantum physical system $\left(U, P_{\mathfrak{I}}, \mathfrak{I}\right)$.

This energy we can represent as integral

$$
\int_{0}^{\infty} \mu_{u_{E}}^{t}\left(d_{t}^{\alpha}\left(\mathfrak{\Im}_{0}^{\alpha}\right)\right) \mathrm{d} t=\int_{0}^{\infty}\left(\int_{\mathfrak{\Im}_{t}^{\alpha}} F_{u_{E}}(\omega) \mathrm{d}\left(\underset{u \in \Re}{\otimes} l_{u}\right)\right) \mathrm{d} t .
$$

We can assume that the measure

$$
\underset{t \in[0, \infty)}{\otimes} \mu_{u_{E}}^{t}\left(\bar{A}_{d_{t}^{\alpha}}\right)=\int_{0}^{\infty}{ }^{\alpha} \mu_{u_{E}}^{t}\left(d_{t}^{\alpha}\left(\Im_{0}^{\alpha}\right)\right) \mathrm{d} t=\int_{0}^{\infty}\left(\int_{\mathfrak{\Im}_{t}^{\alpha}} F_{u_{E}}(\omega) d\left(\underset{u \in \Re}{\otimes} l_{u}\right)\right) \mathrm{d} t
$$

is an analog of the integral action in relation to energy, then the evolution of quantum physical system occurs along trajectories such one-parameter semi groups whose corresponding total energy of evolution is extremal for this action integral. 
If we are interested in evolution of system in interval of time $[0, T]$ then in last integrals we replace symbol $\infty$ by the number $T$.

In closed system does not occur loss or acquisition of energy, therefore for describe evolution of closed quantum physical system we have:

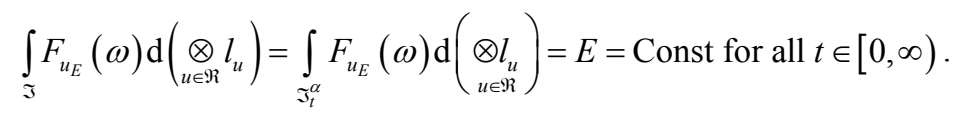

It is clear the extremum principle for integral action in relation to energy in this form for closed system does not give anything. But if we will consider the observable of difference of potential energy and kinetic energy instead observable of the full energy in algebra of quantum system contains, then action integral will be useful.

Consider new another way of describe evolution of closed quantum system.

\section{Entropy in Quantum Physical Systems}

From the second thermodynamic law follows, a closed system has a tendency to move to distribute energy with maximum entropy. The tendency to move to distribute energy with maximum entropy, follows, that the entropy of the distribution energy of the quantum system $\left(U, P_{U}, \mathfrak{J}\right)$, in the moment time $t$, defined so integral

$$
H_{t}^{u}=-\int_{\Im_{t}} \frac{F_{u}(\omega)}{E} \ln \frac{\left(F_{u}\right)(\omega)}{E} \mathrm{~d}\left(\underset{u \in \Re}{\otimes} l_{u}\right)
$$

should increase when increasing $t$.

The entropy $H_{t}^{u}$ will grow if the energy distribution function will be smoothed out when increasing $t$. It will happens if one-parameter semi group $\left\{d_{t}: E_{U} \rightarrow E_{U}, t \in[0, \infty)\right\}$ which describes the movement of closed quantum physical system is a compression semi group, in view, that for every $\varepsilon_{1}>0$ exists $\varepsilon_{2}, 0<\varepsilon_{2} \leq \varepsilon_{1}$, such that if

$$
\omega_{1}, \omega_{2} \in V\left(\omega, u_{E}\right)=\left\{\omega^{\prime} \in \mathfrak{I}|| \omega(u)-\omega^{\prime}(u) \mid \leq \varepsilon_{1}\right\},
$$

then

$$
d_{t}\left(\omega_{1}\right), d_{t}\left(\omega_{2}\right) \in V\left(d_{t}(\omega), u_{E}\right)=\left\{\omega^{\prime} \in \mathfrak{J}|| d_{t}(\omega)\left(u_{E}\right)-\omega^{\prime}\left(u_{E}\right) \mid \leq \varepsilon_{2}\right\},
$$

where parameter $t$ represents time; $u_{E}$ is observable of energy.

Let ${ }^{\alpha} H_{t}^{u}$ be the entropy of quantum system $\left(U, P_{U}, \mathfrak{J}\right)$, in moment $t$ when evolution occurs over a continuous semi group $\left\{d_{t}^{\alpha}: E_{U} \rightarrow E_{U}, t \in[0, \infty)\right\}$.

If we are interested in evolution of system in interval of time $[0, T]$ then in last integrals we replace symbol $\infty$ by the number $T$.

As we see, the extremum principle of action for closed system does not give anything, but here as helping a principle of entropy production maximum, i.e. we must well fine the compression semi group $\left\{d_{t}^{\alpha}: E_{U} \rightarrow E_{U}, t \in[0, T)\right\}$ for which $\frac{\partial\left({ }^{\alpha} H_{t}^{u}\right)}{\partial t}$ is maximal for all $t$. 
If $S_{\alpha}$ is generator of the semi group $\left\{d_{t}^{\alpha}: E_{U} \rightarrow E_{U}, t \in[0, T)\right\}$, then ${ }^{\alpha} H_{t}^{u}$ depends on $S$ as a function ${ }^{\alpha} H_{t}^{u}=H_{t}^{u}\left(S_{\alpha}\right)$. It follows that semi group $\left\{d_{t}^{\alpha}: E_{U} \rightarrow E_{U}, t \in[0, T)\right\}$ for which $\frac{\partial\left({ }^{\alpha} H_{t}^{u}\right)}{\partial t}$ is maximal for all $t$. We can find $S_{\alpha}$ as a solution of the equation $\frac{\partial}{\partial S_{\alpha}} \frac{\partial\left(H_{t}^{u}\left(S_{\alpha}\right)\right)}{\partial t}=0$ by solving it with gradient descent [10].

With increasing entropy, the energy distribution to the quantum system is smoothed out. The energy dispersion band in the states of the system narrows. This causes compressed physical spaces with respect to the energy coordinate, but since the energy of the system does not change, there must happen the tensile propagation along some other coordinates, including coordinates of the corresponding position of the particle. It means that the space where the particles of the quantum system are located expands.

\section{References}

[1] Bogolyubov, H.H., Logunov, A.A., Oksak, A.I. and Todorov, I.T. (1987) General Principles of the Quantum Field Theories. Nauka, Moscow. (In Russian)

[2] Bratteli, O. and Robinson, D. (1982) Operator Algebras and Quantum Statistical Mechanics. Nauka, Moscow. (In Russian)

[3] Griffits, D. (2016) Introduction to Quantum Mechanics. 2nd Edition, Cambridge University Press, Cambridge.

[4] Araki, H. (1999) Mathematical Theory of Quantum Fields. Oxford Science Publication, Oxford.

[5] Gunter, L. (2017) Quantum Mechanics in Matrix Form. Springer, Bremen.

[6] Wolfgang, N. (2017) Theoretical Physics 7, Quantum Mechanics-Methods and Applications. Springer, Berlin.

[7] Mumladze, M. and Zerakidze, Z. (2015) The Criteria of Checking Hypotheses of Quantum States of Quantum Physical System. Journal of Algebra, Number Theory: Advances and Applications, 14, 57-67.

[8] Liusterniik, L.A. and Sobolev, V.I. (1965) Elements of Functional Analyses. Nauka, Moscow. (In Russian)

[9] Bell, J. (2015) Infinite Product Measures. Departments of Mathematics, University of Toronto.

[10] Kantorovich, L.V. and Akilov, G.P. (1984) Functional Analysis. Nauka, Moscow. (In Russian) 